\title{
ANÁLISE DA SUPRESSÃO DA ARBORIZAÇÃO VIÁRIA NA CIDADE DE SÃO CARLOS/SP NO PERÍODO DE 2004 A 2013
}

\author{
ANALYSIS OF THE SUPPRESSION OF STREET TREES IN SÃO CARLOS/SP FROM \\ 2004 TO 2013
}

\author{
Daniel Tonelli Caiche¹, Sandra Regina Mota Silva², Sabrina Mieko Viana ${ }^{3}$, Ricardo Siloto da Silva ${ }^{4}$
}

\section{RESUMO}

A urbanização, notadamente marcada pelo impacto degradador do meio natural, deflagrou diferentes alterações nos territórios. Para reversão de tais condições, o plantio de árvores contribui como uma das possibilidades de qualificação do ambiente urbano, ao proporcionar benefícios, potencializados principalmente pelas árvores de grande porte e em estágio avançado de maturidade. $O$ objetivo desta pesquisa foi analisar a supressão da arborização viária na cidade de São Carlos, Estado de São Paulo, de modo a compreender os fatores que influenciam este processo e assim gerar subsídios para aperfeiçoamento das políticas públicas de planejamento urbano e do sistema de gestão de arborização urbana. Esta análise foi feita com base em dados obtidos na Prefeitura Municipal no período de 2004 a 2013. Foi feita análise de regressão linear simples $(\alpha=0,05)$ para avaliar a significância das variáveis. Os resultados indicaram que as maiores motivações para supressão estavam relacionadas ao conflito com elementos de infraestrutura, em especial o passeio público, seguidos de aspectos "fitossanitários" e na interferência da "mobilidade". Destaca-se que das árvores suprimidas por motivo "fitossanitário", a maioria apresentava a observação "poda inadequada" e somente $10 \%$ das árvores suprimidas estavam mortas. Estes resultados apontam ao planejamento inadequado, que resultaria na incompatibilização do sistema de arborização urbana como o sistema de infraestrutura.

Palavras-chave: Infraestrutura verde; Floresta urbana; Passeio público; Árvores de risco.

\section{ABSTRACT}

The process of urbanization, particularly marked by the degradation in the natural environment brought different changes to the territories. To reverse such situations, the trees can be used as way to improve the environmental quality, potentiated mainly by the positive effects of large and mature trees. This research analyzed the suppression of street trees in São Carlos, São Paulo State, in order to understand which effects are influencing this process and provide support for the improvement of public policies for urban planning. This analysis was carried out by collecting the data produced by City of São Carlos administration, from 2004 to 2013, focusing on the motivations attributed to the suppression of street trees. A simple linear regression $(\alpha=0.05)$ was performed to evaluate the significance of the variables. The results indicate that the major motivations for the tree suppressions are related to conflicts with other infrastructure elements, like the sidewalks, followed by "phytosanitary" aspects and the interference with "mobility". Most of the trees suppressed due to phytosanitary aspects, had an observation of "inadequate pruning" in their register and only one tenth of the trees removed were dead. These results indicate inadequacy in the planning of public pedestrian paths, indicating a space incompatibility with the urban forest and the urban infrastructure systems.

Keywords: Green infrastructure; Urban forest; Sidewalk; Tree Risk.

Recebido em 29.07.2016 e aceito em 31.08.2016

1 Engenheiro Florestal, MSc., Doutorando do Programa de Ciências Ambientais da Universidade Federal de São Carlos. São Carlos, SP Email: dtcaiche@hotmail.com

2 Arquiteta, Dra., Professora do Departamento de Engenharia Civil da Universidade Federal de São Carlos. São Carlos, SP. Email: sandra.mota@ufscar.br

3 Bióloga, Dra., Pós-Doutoranda do Departamento de Engenharia Urbana da Universidade Federal de São Carlos. São Carlos, SP Email: sabrinamieko@gmail.com

4 Arquiteto, Dr., Professor Titular do Departamento de Pós-Graduação em Engenharia Urbana da Universidade Federal de São Carlos. São Carlos, SP. Email: rss@ufscar.br 


\section{INTRODUÇÃO}

O conjunto de mudanças no processo de produção de bens observadas a partir da chamada Revolução Industrial teve um profundo impacto nas estruturas sociais e na ocupação e organização dos territórios. Iniciada na Inglaterra em meados do século XVIII expandiu-se pelo mundo a partir do século XIX, tendo como marca o aumento e crescimento das indústrias (PAULO, 2010). Em decorrência deste processo, no Brasil, durante o século XX, ocorreu um intenso fenômeno de urbanização junto com o crescimento demográfico urbano. A população brasileira entre 1940 e 2010 cresceu aproximadamente 4,5 vezes, enquanto que, no mesmo período, a população urbana teve um crescimento de aproximadamente 12 vezes. Atualmente aproximadamente $85 \%$ da população brasileira é urbana, sendo que o Estado de São Paulo apresenta-se como o $3^{\circ}$ estado mais urbanizado do país, com uma população urbana de aproximada 96\% (IBGE, 2010a).

Uma das diversas definições para o conceito de urbanização descreve este processo como resultado da "concentração de uma população, a partir de certos limites de dimensão e intensidade" (CASTELLS, 1983, p. 39). A materialização desigual deste processo fomentou a consolidação de carências generalizadas, nos precários assentamentos das regiões periféricas e interstícios urbanos. Foram gerados impactos sociais e ambientais, nos quais se identifica uma interação entre os conflitos, que potencializa a ocorrência de problemas, na disputa pelos escassos espaços disponíveis (DOWBOR, 2007). A vegetação em áreas urbanas, com o tempo foi se perdendo. Ainda hoje, o processo de urbanização, se inicia a partir da limpeza do terreno, ou seja, da remoção da cobertura vegetal local, quando existente.

Deste cenário desdobra-se uma série de consequências negativas para o habitat local, de ordem ecológica, econômica e social. Pode-se citar como exemplos: erosão dos solos, assoreamento de corpos hídricos, eutrofização de lagos e lagoas, aumento do escoamento superficial, ilhas de calor, aumento no consumo energético com fins térmicos, redução da umidade relativa e da qualidade do ar, degradação da paisagem, entre outros (NOWAK, 2006; SOARES et al., 2010; SEGARAN et al., 2014; HSIE et al., 2016; LAYMAN et al., 2016).

Neste contexto, o uso do elemento arbóreo nas áreas urbanizadas configura-se como uma das estratégias com potencial de melhoria da qualidade de vida de seus citadinos. $O$ planejamento do elemento arbóreo se apresenta em múltiplas possibilidades nos espaços públicos e privados (WICKHAM, 2010). Dentro do leque de áreas públicas passíveis de arborização será destacado neste trabalho o passeio, um dos maiores espaços públicos das cidades, quando considerada área superficial. Nestes espaços ocorrem em simultâneo os deslocamentos de pessoas e mercadorias, as trocas sociais e culturais, tendo como base a infraestrutura urbana com a implantação da arborização. 
A vegetação arbórea, ao longo da malha urbana, constitui-se, assim, em uma potente ferramenta que pode auxiliar na preservação do equilíbrio biológico, exercendo as funções de zonas tampão de fragmentos remanescentes, além de estrategicamente servir de trampolins ecológicos, facilitando o fluxo gênico de fauna e flora (KATTEL et al., 2013).

A arborização urbana pode não só melhorar a qualidade ambiental de uma localidade, mas também promover um grande impacto social na saúde, na educação, no trabalho e na segurança, ao proporcionar condições climáticas e paisagísticas, tornando locais mais atrativos que contribuem para uma melhor qualidade de vida e servem, entre outros, para o lazer e para as atividades físicas (BOONE et al., 2012).

Desta maneira, o uso do elemento arbóreo no planejamento e na produção das cidades está diretamente ligado à qualidade de vida no ambiente urbano - seja pelas condições ecológicas, climáticas, econômicas, ou pela maneira de se perceber a cidade, com benefícios para a psique representando um universo de potencialidades sinergéticas. (VELARDE et al., 2007; WOLFE; MENNIS, 2012).

No entanto, historicamente, o sistema de arborização das cidades brasileiras tem sido marcado por um planejamento que resulta em um cenário de conflitos com partes integrantes dos sistemas de infraestrutura urbana e que se desdobra, entre outros problemas, na baixa qualidade ambiental do espaço público urbano (HERZOG, 2010). A disputa acirrada por espaço envolvendo as árvores e os demais equipamentos urbanos, como postes, tubulações, fiação elétrica, edificações e afins, conduz a construção de paisagens cada vez mais cinzas, impermeáveis e de baixa qualidade.

A fim de aportar elementos que possam ser usados para o adequado planejamento e gestão da arborização da cidade de São Carlos, esta pesquisa teve como objetivo avaliar os motivos geradores da supressão de indivíduos arbóreos localizados no sistema viário.

\section{MATERIAIS E MÉTODOS}

O município de São Carlos está localizado no estado de São Paulo entre a latitude 22ํㅡㄴ 01' 03" S e longitude 47 53' 27" O, possui área total corresponde atualmente a 1.140,92 km², dos quais $80,8 \mathrm{~km}^{2}$ correspondem à área urbana (7\% do total), com população total de 221.250 habitantes, dos quais $96 \%$ dos domicílios estão em territórios urbanizados (IBGE, 2010a). Destes domicílios urbanos, 89,5\% são considerados "arborizados" (IBGE, 2010b). Embora este número, no primeiro momento, indique um valor representativo de residências dotadas de indivíduos arbóreos, este indicador, levantado pela primeira vez, pelo IBGE, no censo de 2010, deve ser avaliado com maior cuidado porque utiliza como critério apenas a presença de uma 
árvore por face de quarteirão, assim todas as residências, de ambos os lados da via, serão consideradas arborizadas, caso exista apenas uma árvore no local.

Para analisar este cenário urbano em que a supressão dos indivíduos arbóreos é o tratamento cultural mais severo que pode ser executado, e diante da escassez de trabalhos que abordem esse tema na arborização viária, foi elaborada uma estrutura de trabalho adaptada que se baseou nos trabalhos de inventário e levantamentos de Silva Filho et al. (2002), Volpe-Filik (2007) e Sucomine e Sales (2010), assim como a pesquisa realizada por Martelli e Barbosa-Junior (2011).

Desta maneira, a pesquisa foi estruturada em três fases distintas e complementares: Revisão da literatura, coleta, tabulação e diagramação dos dados e análise dos resultados. Na etapa de coleta, foi realizada uma consulta exploratória à base de dados ambientais municipais da Prefeitura Municipal de São Carlos. Foram analisados os Laudos Técnicos de Vegetação emitidos no período de 2004 a 2013. Para ser incorporado ao universo amostral da pesquisa, o documento técnico supracitado deveria contemplar as seguintes condições: supressão de indivíduo arbóreo; estar inserido no perímetro urbano e localizado no espaço público denominado passeio. Uma vez inserido no universo amostral, as variáveis observadas em cada laudo técnico foram os motivos que embasaram a atividade de supressão. Assim, as motivações foram divididas em quatro temas estruturais, para melhor análise e compressão: Infraestrutura; Indivíduo; Construtivo; Diversos.

O tema 1 "Interferência com a infraestrutura" compõe-se das supressões motivadas por conflitos com as diversas redes de infraestrutura urbana: mobilidade (placas, postes, semáforos, obstáculos para pedestres e automóveis nos passeios públicos); abastecimento de água, coleta de esgoto e águas pluviais (tubulações subterrâneas, hidrantes e tubulações superficiais); sistema de abastecimento de energia e comunicação (fiação aérea de distribuição de energia, telefone, televisão a cabo).

Já o tema 2 "Problemas estruturais da árvore", considera as motivações diretamente relacionadas às condições biológicas, fisiológicas e estruturais dos indivíduos suprimidos, como: condições fitossanitárias (incidência de insetos xilófagos, doenças e fungos decompositores), inclui-se nesta categoria os problemas derivados de podas inadequadas; inclinação e ou situações que comprometam a estabilidade dos exemplares e à condição de seca/morta.

O tema 3 "Afetação a edificações" refere-se às supressões arbóreas motivadas pelos danos causados em estruturas construtivas na disputa de espaço com o sistema radicular dos exemplares, danos aos muros, garagens das edificações, bem como danos às próprias edificações, nos casos de ampliação ou reformas; Enquanto que o tema 4 "Diversos" inclui as 
diversas motivações não classificadas que apareceram durante a análise, como: anelamentos, segurança; morcegos, queimadas entre outros.

\section{RESULTADOS E DISCUSSÃO}

Foram tabulados dados referentes à 2.146 árvores suprimidas, pertencentes a 39 famílias e 95 espécies, durante o recorte temporal de análise (2004-2013). Destas, a espécie com maior número de exemplares suprimidos (705 exemplares) foi a Schinus molle $L$, seguida da Magnolia champaca (L.) Baill. ex Pierre (264 exemplares), Ficus benjamina L (227 exemplares), Caesalpinia pluviosa DC. var peltophoroides Benth (115 exemplares) e Terminalia catappa $L$ (95 exemplares) que, em conjunto, representam o 65,52\% das árvores cortadas.

A primeira análise realizada consistiu na distribuição dos casos de supressão de indivíduos arbóreos ao longo do período histórico analisado. As supressões foram somadas a cada ano, com intuito de observar se há um padrão de distribuição ou alguma tendência presente para o período. Os dados podem ser observados na Figura 1.

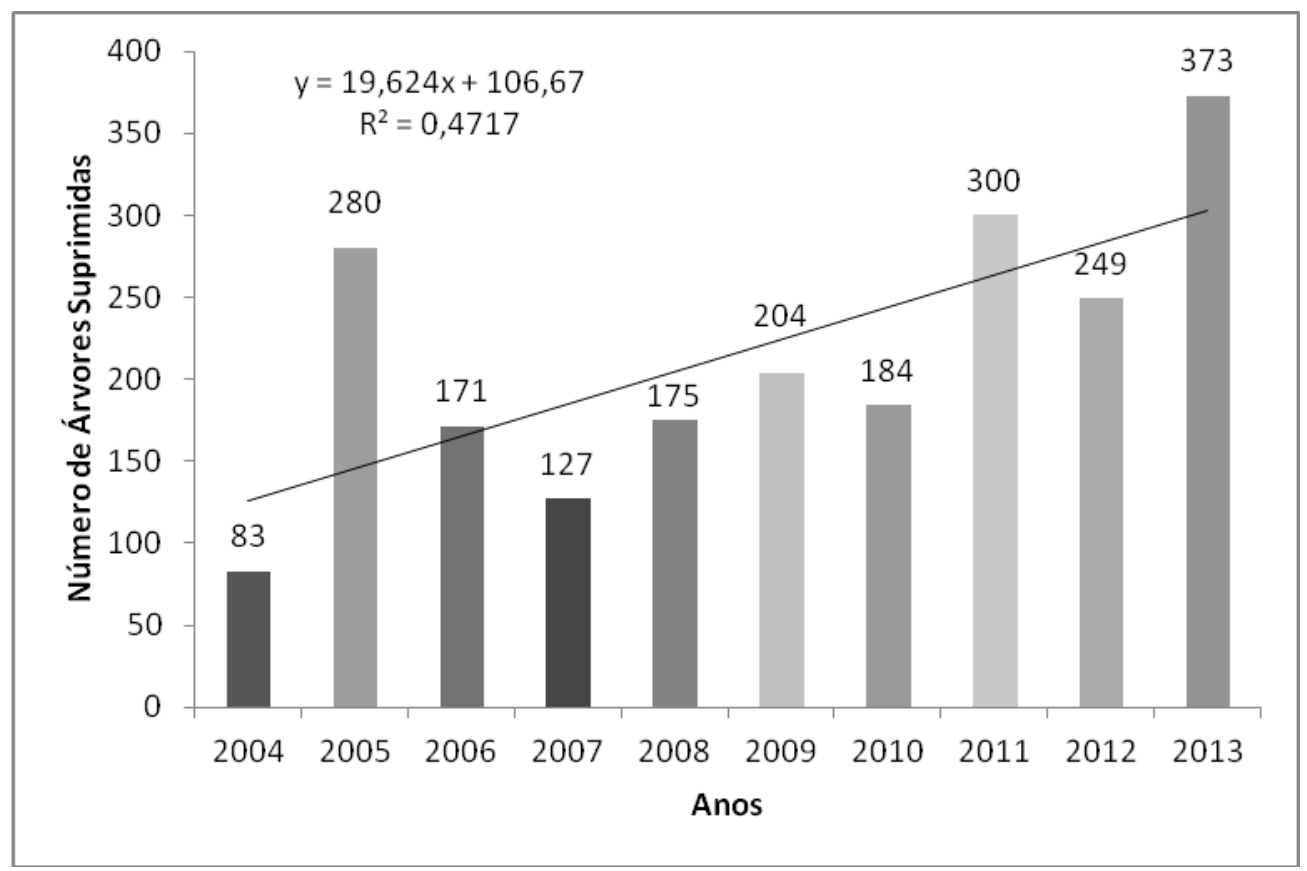

Figura 1. Número de supressões arbóreas por ano no município de São Carlos-SP para o período 2004 $-2013$

Figure 1. Number of tree suppression per year in the municipality of São Carlos-SP from 2004 to 2013

Nota-se uma tendência crescente na quantidade de supressões arbóreas ao longo dos anos no período analisado. Excluindo-se o ano de 2005, os últimos três anos (2011, 2012 e 
2013) registraram maior número de cortes. A análise de regressão linear simples indicou a variável 'Ano' como estatisticamente significativa $(\alpha=0,05)$, o que aponta uma taxa de aumento no corte de árvores em função do tempo, que de acordo com o modelo da regressão linear, com estimativa de 19,6 indivíduos a cada ano.

A seguir, serão descritos os dados de supressão arbórea em relação aos fatores motivadores dos cortes, segundo os laudos analisados. Vale ressaltar que a supressão de um mesmo indivíduo poderia ser descrita por mais de um motivo. Desta forma, os dados apresentados na Tabela 1 totalizam número superior ao total de supressões analisadas.

Tabela 1. Número de supressões arbóreas distribuídas em relação à motivação

Table 1. Number of tree suppressions distributed in relation to motivation

\begin{tabular}{llcc}
\hline & Motivo & Supressões & Frequência (\%) \\
& Passeio/canteiro & 983 & 26,9 \\
Interferência com a & Água/Esgoto/Gás & 254 & 6,9 \\
infraestrutura (tema 1) & Fiação aérea & 244 & 6,7 \\
& Mobilidade & 418 & 11,4 \\
& Subtotal & 1.899 & 51,9 \\
\hline \multirow{4}{*}{ Problemas estruturais da } & Fitossanitário & 461 & 21,5 \\
árvore (tema 2) & Inclinação/Estrutural & 343 & 16,0 \\
& Morta/Seca & 226 & 10,5 \\
& Porte Incompatível & 216 & 10,1 \\
& Subtotal & 1.246 & 34,0 \\
\hline \multirow{2}{*}{ Afetação a edificações } & Ampliação/Reforma & 26 & 1,2 \\
(tema 3) & Garagem & 146 & 6,8 \\
& Muro/Edificação & 310 & 14,4 \\
& Subtotal & 482 & 13,2 \\
\hline \multirow{2}{*}{ Diversos (tema 4) } & Diversos & 32 & 1,5 \\
& Subtotal & 32 & 0,9 \\
\hline & TOTAL & $\mathbf{3 . 6 5 9}$ & $\mathbf{1 0 0 \%}$ \\
\hline
\end{tabular}

Por meio da comparação entre os temas, a magnitude das supressões motivadas por conflito com elementos de infraestrutura soma mais da metade $(51,9 \%)$ das motivações que levaram ao corte. Dentro deste tema, aproximadamente $26,9 \%$ das supressões no período (983 exemplares) envolviam conflitos entre os passeios públicos e a arborização viária, onde foram considerados os indivíduos removidos com a justificativa de estarem causando danos estruturais à pavimentação do passeio público, o que coincidia de forma recorrente com o 
subdimensionamento do canteiro. Estudos já realizados sobre este tema, que abordaram o tamanho da área livre de pavimentação deixada na base das árvores e os danos às calçadas, indica correlação entre estes fatores (VOLPE-FILIK et al., 2007). Em um levantamento realizado em 2010 , foi detectado que $76 \%$ das árvores presentes na região central do município de São Carlos-SP tinham pouca ou nenhuma área livre no entorno de sua base (SUCOMINE; SALES, 2010). Desta maneira, é de extrema importância buscar a ampliação os canteiros para uma melhor interação entre a planta e o ambiente no entorno, possibilitando a diminuição de problemas com calçadas.

O segundo tema com maior número de supressões corresponde a "Problemas estruturais da árvore", em que o motivo "fitossanitário" foi o mais representativo com 461 $(12,6 \%)$ supressões associadas com esse problema, com relação direta à saúde do exemplar. Desse total, 250 (7,3\%) continham a observação de "poda inadequada".

A terceira e a quarta motivações que justificaram mais números de supressões foram "mobilidade" (11,4\%) e "inclinação" (9,4\%), ambas inseridas nos temas "Interferência com a infraestrutura" e "Problemas estruturais da árvore", respetivamente. Ainda no tema 1, foram observadas $244(6,7 \%)$ supressões devido ao conflito com o sistema de transmissão de energia e telecomunicação, por meio dos cabeamentos aéreos que normalmente estão no alinhamento dos canteiros destinados à arborização dos passeios.

No tema 3 (Afetação a edificações) a motivação "ampliação/reforma" aparece na comparação total como a sexta motivação maior número de supressões com 310 (8,5\%) devido ao conflito das árvores com as estruturas dos muros e das próprias edificações, tanto comerciais quanto residenciais.

Outra constatação de grande importância para compreensão do cenário da arborização urbana do município é que do total analisado, apenas $226(6,2 \%)$ das supressões autorizadas estavam mortos e/ou secos. Contudo, ressalta-se que nestes casos pode haver relação entre algum dos outros motivos de supressão, como conflito com elemento de infraestrutura ou devido a alguma ação inadequada de manejo, que tenha sido a causa mortis destes exemplares. Além disso, este fato indica que a maioria das árvores presentes na arborização da cidade, são cortadas prematuramente antes que completem seu ciclo vital. Assim, infere-se que poucos exemplares arbóreos do sistema viário conseguem chegar à maturidade, reduzindo todo o potencial dos benefícios já conhecidos relacionados à arborização deixam de ser sentidos pela comunidade. Em vez de propiciar efeitos positivos, em muitos casos, ao se considerar os custos de implantação, manutenção e supressão de indivíduos jovens, pode-se chegar ao resultado de uma relação negativa entre investimentos financeiros aportados e serviços ecossistêmicos prestados (LYYTIMÄKI; SIPILÄ; 2009) 
Os indivíduos agrupados na categoria "Diversos" foram citados em 32 casos de supressão que representam apenas $0,87 \%$ do total das motivações. A Figura 2 descreve quais foram estes motivos, menos frequentes, porém importantes para uma compreensão completa deste aspecto.

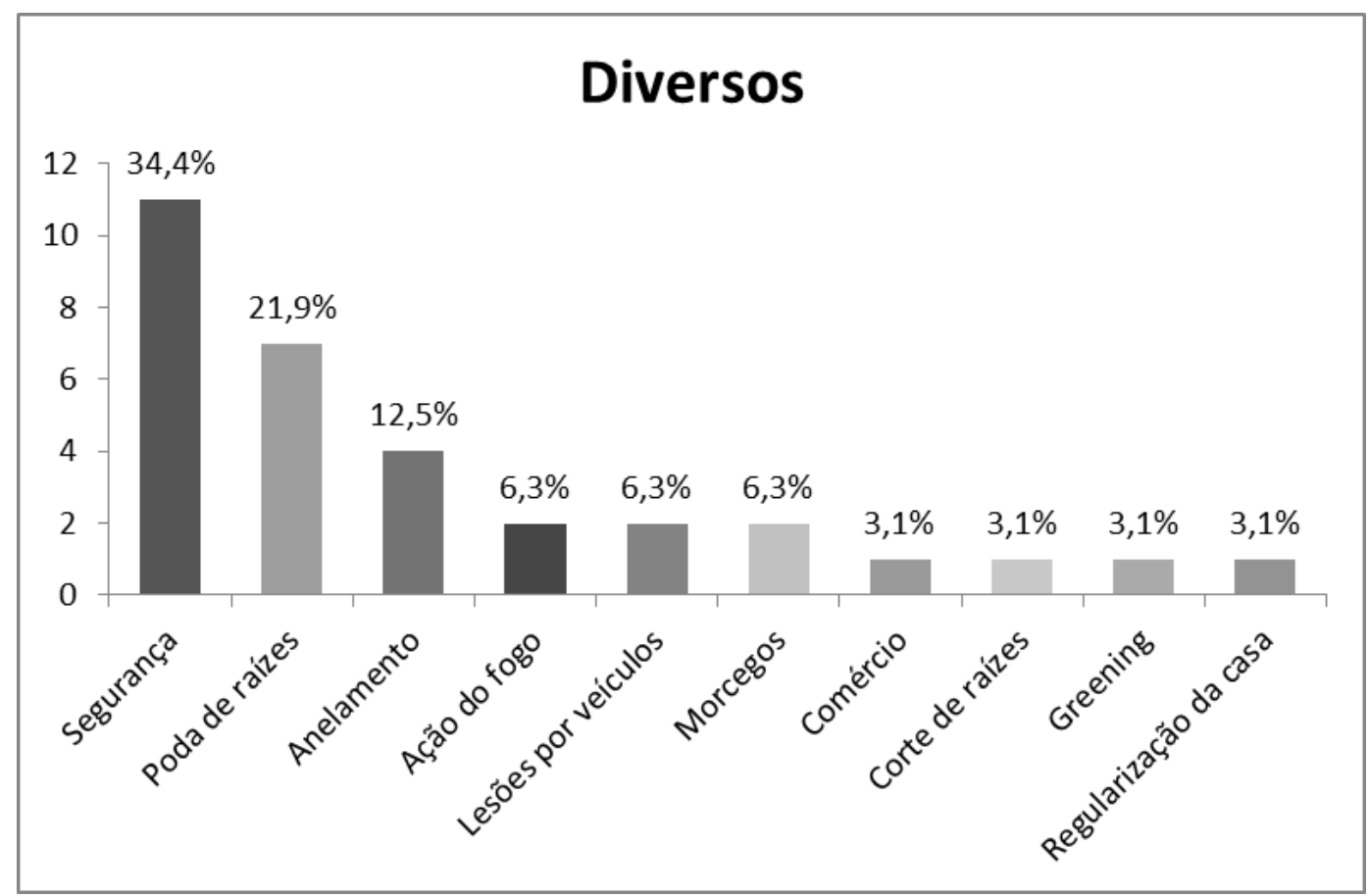

Figura 2. Supressões associadas aos motivos classificados como "diversos"

Figure 2. Suppressions associated with reasons classified as "various"

Nesta última categoria, destaca-se a poda de raízes, com $22 \%$ dos casos. Pode-se inferir que este número de ocorrências pode estar ligado ao elevado número de ocorrências do conflito entre sistema radicular e canteiro, como já indicado.

O item anelamento (atividade de retirada do câmbio vascular em anel com objetivo de secar o exemplar arbóreo), em 13\% dos casos do tema Diversos, demonstra a distância e alienação dos cidadãos em relação aos serviços e benefícios que os exemplares arbóreos proporcionam. Também é notável que $6 \%$ dos exemplares suprimidos contidos no tema 'Diversos' tiveram como motivação da supressão o fato de terem sido atingidas por automóveis, principalmente caminhões ou veículos de grande porte.

De maneira geral, houve uma tendência crescente no número de exemplares suprimidos ao longo do tempo. Como possibilidades de explicação para esta observação podese citar o aumento no número de laudos emitidos como consequência do ao aumento da demanda de vistorias técnicas sobre as árvores nos passeios por parte da população. Outra possibilidade consiste na crescente atenção para com o tema arborização urbana, que reflete no aumento das supressões legais, autorizadas pela municipalidade. 
O conflito referente à localização do exemplar e sua relação com o ambiente construído, quando não planejado ou com planejamento inadequado, na maioria das vezes é fatal para o exemplar arbóreo, já que, pelas práticas culturais predominantes, não se desconstrói e demole edificações para preservação de árvores. Este fato também foi observado no estudo de Martelli e Barbosa Junior (2011).

Diante deste quadro, pode-se inferir que os órgãos responsáveis tanto pelo planejamento quanto pela aprovação e gestão desta infraestrutura verde (arborização viária) produzem espaços que criam dificuldades para crescimento sadio de exemplares arbóreos. Resultado deste cenário, o número de supressões relacionadas às atividades de manejo e poda, destacou-se um efeito colateral de grande relevância para a gestão do sistema de arborização. A execução desta atividade de forma inadequada gera um grande impacto na exposição do indivíduo arbóreo, que ao não conseguir compartimentalizar os danos causados pelo manejo inadequado fica exposto a incidência de insetos xilófagos e fungos apodrecedores, que podem determinar a supressão deste indivíduo (BRAZOLIN et al., 2014).

Para se mitigar este cenário de conflitos, a arborização urbana deveria ser incluída no mesmo grau de prioridade no processo de concepção e gestão do espaço urbano, quando comparada com as demais temáticas de infraestrutura (abastecimento de água, de energia e esgotamento sanitário) a fim de se criar um espaço compatível com as árvores das diferentes espécies e reduzindo o número de intervenções de manejo (poda).

Os sistemas de infraestrutura urbana devem evoluir de projetos setoriais para abordagens integradas de planejamento que compatibilizem os espaços do conjunto de passeios públicos, que compõe e interligam o sistema de espaços livres de uma cidade.

Desta maneira, reconhece-se que há um amplo horizonte de potencialidades e possibilidades viáveis para melhoria dos passeios públicos, por meio do uso racional do espaço e que necessariamente inclua a questão arbórea. No entanto, entende-se que há um longo caminho a percorrer nesta direção, que passa pela mudança de prioridades no modus operandi e na forma fragmentada de divisão de responsabilidades e atribuições para cada setor envolvido.

\section{CONCLUSÕES}

A maioria das supressões de árvores autorizadas durante o período avaliado não correspondem a árvores mortas ou secas que terminaram seu ciclo vital, e sim foram motivadas por conflitos com outros elementos de infraestrutura urbana que compõe o passeio público. Estas situações decorrem do planejamento deficitário e que demonstram a principal 
falha encontra-se na atividade de concepção dos projetos de arborização inseridos nos passeios públicos.

As autorizações de supressão devidas aos itens 'Problemas estruturais da árvore' e 'Afetação a edificações', constituem a segunda e terceira causa de corte das árvores durante o período avaliado, sendo que, no caso do primeiro, foi observada uma forte relação entre as podas inadequadas e o motivo de supressão relacionado a aspectos fitossanitários.

\section{REFERÊNCIAS}

BOONE, C.G.; COOK, E.; HALL, S.J; NATION, M.L.; GRIMM, N. B.; RAISH, C.B.; FINCH, D.M.; YORK, A.M. A comparative gradient approach as a tool for understanding and managing urban ecosystems. Urban Ecosystems, New York, v. 15, p.795-807, 2012.

BRAZOLIN, S.; TOMAZELLO FILHO, M.; YOJO, T.; OLIVERA NETO, M.A.; ALBUQUERQUE, A.R.; SETTE JÚNIOR, C.R. Propriedades físico-mecânicas do lenho deteriorado por fungos apodrecedores de árvores de Tipuana tipu. CERNE, Lavras, v.20, n.2, p.183-190, 2014.

CASTELLS, M. A questão urbana. Rio de Janeiro: Paz e Terra, 1983.

DOWBOR, L. Inovação social e sustentabilidade. Revista Brasileira de Gestão Urbana, Curitiba, v. 1, n. 1, p. 109-125, 2009.

HERZOG. C, P. Infraestrutura Verde: Sustentabilidade e Resiliência para a Paisagem Urbana. Revista LabVerde, São Paulo, v. 1, n. 1, 2010.

HSIE, C.; JAN, F.; ZHANG, L. A simplified assessment of how tree allocation, wind environment, and shading affect human comfort. Urban Forestry \& Urban Greening, Amsterdam, v.18, p.126-137, 2016.

IBGE. Instituto Brasileiro de Geografia e Estatística. Séries Históricas. 2010a. Disponível em: $<$ http://seriesestatisticas.ibge.gov.br/series.aspx?no=1\&op=2\&vcodigo=POP100\&t=populacao-residentedistribuicao-grandes-regioes >. Acesso em: 24 ago. 2016.

IBGE. Instituto Brasileiro de Geografia e Estatística. Censo Demográfico 2010: Características urbanísticas do entorno dos domicílios - Tabelas 2010b. Disponível em: <http://www.ibge.gov.br/home/estatistica/populacao/censo2010/entorno/entorno_tab_municipios_zip_xls. shtm>. Acesso em 26 ago. 2016.

KATTEL, G.; ELKADI, H.; MEIKLE, H. Developing a complementary framework for urban ecology. Urban Forestry \& Urban Greening, Amsterdam, v.12, n. 4, p.498-508, 2013.

LAYMAN, R.M.; DAY. S.D.; MITCHELL, D.K.; CHEN, Y.; HARRIS, J. R.; DANIELS, W.L. Below ground matters: Urban soil rehabilitation increases tree canopy and speeds establishment. Urban Forestry \& Urban Greening, Amsterdam, v.16, p.25-35, 2016.

LYYTIMÄKI, J.; SIPILÄ, M. Hopping in one leg - The challenge of ecosystems disservices for urban green management. Urban Forestry \& Urban Greening, Amsterdam, v.8, n.4, p.309-315, 2009.

MARTELLI, A.; BARBOSA JUNIOR, J. Incidência e fatores causais das supressões da arborização urbana no município de Itapira, Estado de São Paulo. Revista Acadêmica: Ciências Agrarias e Ambientais, Curitiba, v.9, n. 2, p.215-222, 2011.

MILANO, M. S.; DALCIN, E. C. Arborização de vias públicas. Rio de Janeiro: Light, 2000. 226 p. 
NOWAK, D. Institutionalizing urban forestry as a "biotechnology" to improve environmental quality. Urban Forestry \& Urban Greening, Amsterdam, v. 5, n.2, p.93-100, 2006.

OLIVEIRA, C.H. Planejamento ambiental na cidade de São Carlos (SP) com ênfase nas áreas públicas e áreas verdes: diagnóstico e propostas. 181p.Dissertação (Mestrado em Ecologia e Recursos Naturais) - Universidade Federal de São Carlos, São Carlos, 1996.

PAULO, R. R. O desenvolvimento industrial e o crescimento populacional como fatores geradores do impacto ambiental. Veredas do Direito, Belo Horizonte, v.7, n.13/14, p.173-189, 2010.

SEGARAN, S.R.R.; LEWIS, M.; OSTENDORF, B. Stormwater quality improvement potential of an urbanised catchment using water sensitive retrofits into public parks. Urban Forestry \& Urban Greening, Amsterdam, v.13, n. 2, p.315-324, 2014.

SILVA FILHO, D.F.; PIZETTA, P.U.C.; ALMEIDA, J.B.S.A.; PIVETTA, K.F.L.; FERRAUDO, A.S. Banco de dados relacional para cadastro, avaliação e manejo da arborização em vias públicas. Revista Árvore, Viçosa, v.26, n.5, p.629-642, 2002.

SOARES, A.L.; REGO, F.C., McPHERSON, E.G.; SIMPSON, J.R., PEPER,P.J, IAO, Q. Benefits and costs of street trees in Lisbon, Portugal. Urban Forestry \& Urban Greening, Amsterdam, v.10, n. 2, p.69-78, 2010.

SUCOMINE, N. M.; SALES, A. Caracterização e Análise do Patrimônio Arbóreo da malha viária urbana central do município de São Carlos-SP. Revista da Sociedade Brasileira de Arborização Urbana, Piracicaba, v. 5, n. 4, p. 128-140, 2010.

VELARDE, M.D.; FRY, G.; TVEIT, M. Health effects of viewing landscapes - Landscape types in environmental psychology. Urban Forestry \& Urban Greening, Amsterdam, v.6, n. 4, p.199-212, 2007.

VOLPE-FILIK, A.; SILVA, L. F.; LIMA, A. M. P. Avaliação da arborização de ruas do bairro São Dimas na cidade de Piracicaba-SP através de parâmetros qualitativos. Revista da Sociedade Brasileira de Arborização Urbana, Piracicaba, v. 2, n. 1, p. 34-43, 2007.

WICKHAM, J. D.; RIITTERS, K., H. WADE, T. G.; VOGT, P. A national assessment of green infrastructure and change for the conterminous United States using morphological image processing. Landscape and Urban Planning, Amsterdam, v. 94, p.186-195, 2010.

WOLFE, M. K.; MENNIS, J. Does vegetation encourage or suppress urban crime? Evidence from Philadelphia, PA. Landscape and Urban Planning, Amsterdam, v. 108, n. 2/4, p. 112-122, 2012. 